\title{
Criminologie
}

\section{Les droits procéduraux des victimes devant la Cour pénale internationale}

\section{Gilbert Bitti}

Volume 44, numéro 2, automne 2011

Les droits des victimes dans un contexte international

URI : https://id.erudit.org/iderudit/1005792ar

DOI : https://doi.org/10.7202/1005792ar

Aller au sommaire du numéro

Éditeur(s)

Les Presses de l’Université de Montréal

ISSN

0316-0041 (imprimé)

1492-1367 (numérique)

Découvrir la revue

Citer cet article

Bitti, G. (2011). Les droits procéduraux des victimes devant la Cour pénale internationale. Criminologie, 44(2), 63-98. https://doi.org/10.7202/1005792ar
Résumé de l'article

Si l'instauration de droits procéduraux pour les victimes devant la Cour pénale internationale a constitué sans aucun doute une innovation fondamentale par rapport aux deux tribunaux ad hoc pour l'ex-Yougoslavie et le Rwanda, cette innovation se caractérise par son imprécision. Dans cet article nous étudierons le droit des victimes à participer aux procédures. On étudiera d'abord les droits procéduraux des victimes de la situation, puis les droits procéduraux des victimes de l'affaire. Nous conclurons que la mise en oeuvre de la participation des victimes par la jurisprudence leur laisse encore un sentiment d'incertitude. La solution pour une meilleure intégration des victimes à la procédure devant la CPI devrait passer par une codification de leurs droits. 


\title{
Les droits procéduraux des victimes devant la Cour pénale internationale
}

\author{
Gilbert Bitti \\ Conseiller juridique hors classe \\ Section préliminaire de la Cour pénale internationale \\ gilbert.bitti@icc-cpi.int
}

RÉSUMÉ • Si l'instauration de droits procéduraux pour les victimes devant la Cour pénale internationale a constitué sans aucun doute une innovation fondamentale par rapport aux deux tribunaux ad hoc pour l'ex-Yougoslavie et le Rwanda, cette innovation se caractérise par son imprécision. Dans cet article nous étudierons le droit des victimes à participer aux procédures. On étudiera d'abord les droits procéduraux des victimes de la situation, puis les droits procéduraux des victimes de l'affaire. Nous conclurons que la mise en œuvre de la participation des victimes par la jurisprudence leur laisse encore un sentiment d'incertitude. La solution pour une meilleure intégration des victimes à la procédure devant la CPI devrait passer par une codification de leurs droits.

MOTS-CLÉS - Droits des victimes, participation, Cour pénale internationale, droit international, contrôle judiciaire, pouvoir discrétionnaire du procureur.

\section{Introduction $^{1}$}

Si l'instauration de droits procéduraux pour les victimes devant la Cour pénale internationale (ci-après «la Cour» ou «la CPI») a constitué sans aucun doute une innovation fondamentale par rapport aux deux tribunaux ad hoc pour l'ex-Yougoslavie et le Rwanda, cette innovation se caractérise par son imprécision. En effet, le texte principal en la matière, à savoir l'article 68, paragraphe 3 du Statut de Rome ${ }^{2}$ (ci-après «le

1. Les points de vue exprimés dans le présent document sont ceux de l'auteur en sa capacité personnelle et ne reflètent en aucune façon ceux de la Cour pénale internationale.

2. Adopté à Rome le 17 juillet 1998, document A/CONF.183/9, amendé dans sa version française par les procès-verbaux des 10 novembre 1998, 12 juillet et 30 novembre

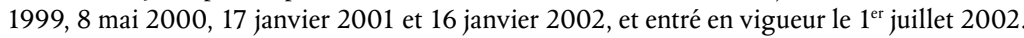

Criminologie, vol. $44, \mathrm{n}^{\circ} 2$ (2011) 
Statut») de la CPI, se contente de donner aux victimes un droit général de participation à la procédure devant la Cour sans en préciser le contenu:

Lorsque les intérêts personnels des victimes sont concernés, la Cour permet que leurs vues et préoccupations soient exposées et examinées, à des stades de la procédure qu'elle estime appropriés et d'une manière qui n'est ni préjudiciable ni contraire aux droits de la défense et aux exigences d'un procès équitable et impartial. Ces vues et préoccupations peuvent être exposées par les représentants légaux des victimes lorsque la Cour l'estime approprié, conformément au Règlement de procédure et de preuve.

Ce texte soulève plusieurs questions: qui sont des victimes? Quand peut-on considérer que leurs intérêts personnels sont concernés? À quels stades peuvent-elles participer et en quoi consiste cette participation? Le Règlement de procédure et de preuve ${ }^{3}$ (ci-après «le Règlement») n'apporte pas beaucoup plus de précisions sur ce qu'il faut entendre par «vues et préoccupations» et la jurisprudence en a conclu qu'il revenait à chaque chambre de la Cour de fixer le contenu des droits procéduraux des victimes ${ }^{4}$.

S'agissant des droits procéduraux des victimes, la jurisprudence a très vite distingué entre les «victimes d'une situation» et les «victimes d'une affaire»:

La Chambre considère que le Statut, le Règlement de procédure et de preuve et le Règlement de la Cour envisagent la distinction entre situations et affaires comme faisant l'objet de procédures distinctes engagées par l'un quelconque des organes de la Cour. Les situations, généralement définies par des paramètres temporels, territoriaux et éventuellement personnels, telle que la situation sur le territoire de la République démocratique du

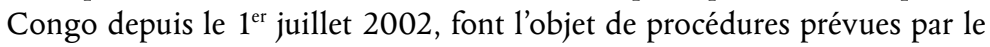
Statut afin de décider si une situation donnée doit faire l'objet d'une enquête pénale, et de l'enquête en tant que telle. Les affaires, comprenant des incidents spécifiques au cours desquels un ou plusieurs crimes de la compétence de la Cour semblent avoir été commis par un ou plusieurs

3. Adopté par l'Assemblée des États Parties au Statut lors de sa première session, New York, 3-10 septembre 2002, document ICC-ASP/1/3.

4. CPI, Situation en République démocratique du Congo, Affaire le Procureur c. Germain Katanga et Matbieu Ngudjolo Chui, ICC-01/04-01/07-1788, Chambre de première instance II, Décision relative aux modalités de participation des victimes au stade des débats sur le fond, 22 janvier 2010, paragraphes 53 et 54 . 
suspects identifiés, font l'objet de procédures qui ont lieu après la délivrance d'un mandat d'arrêt ou d'une citation à comparaître .

Les victimes d'une situation correspondent donc à toutes les victimes de crimes qui ne sont pas, ou pas encore, poursuivis par le procureur de la CPI, mais qui relèvent de la compétence de la Cour dans une situation donnée, à savoir par exemple toutes les victimes de génocide, de crimes contre l'humanité et de crimes de guerre commis en République démocratique du Congo (RDC) depuis le $1^{\text {er }}$ juillet 2002.

En revanche, les victimes d'une affaire sont celles qui ont souffert un préjudice du fait des crimes poursuivis par le procureur de la CPI dans une affaire particulière: ainsi les victimes de l'affaire Katanga et Ngudjolo Chui, au sein de la situation en RDC, se limitent à celles qui ont souffert un préjudice du fait d'une seule attaque commise en un seul jour (le 24 février 2003) et sur un seul village, à savoir celui de Bogoro. C'est dire si les victimes d'une affaire particulière constituent un groupe infiniment plus restreint que celui des victimes d'une situation.

Dans cet article nous étudierons les décisions prises par les différentes chambres de la CPI pour comprendre ce qui est advenu de cette innovation fondamentale que constituait en 1998 leur droit de participer à la procédure devant la CPI. La participation des victimes à la procédure a fait l'objet de débats difficiles à la CPI depuis les premiers pas de celle-ci et une multitude de décisions ont été prises par les différentes chambres de la Cour à ce sujet qu'il nous faudra étudier. Suivant la jurisprudence, nous distinguerons entre les victimes de la situation et les victimes d'une affaire. Ensuite nous suivrons la logique du déroulement de la procédure devant la CPI en commençant avec la participation des victimes au stade préliminaire des affaires, puis au stade du procès lui-même.

\section{Les droits procéduraux des victimes de la situation}

À défaut de définition précise des droits procéduraux des victimes, les chambres préliminaires ont tenté d'établir chacune un «système de participation» pour ces victimes. Ces systèmes n'ont pour l'instant abouti à aucune mise en œuvre pratique pour celles-ci et les droits

5. CPI, Situation en République démocratique du Congo, ICC-01/04-101, Chambre préliminaire I, Décision sur les demandes de participation à la procédure de VPRS 1, VPRS 2, VPRS 3, VPRS 4, VPRS 5 et VPRS 6, 17 janvier 2006. 
procéduraux des victimes de la situation sont demeurés largement théoriques.

\section{Les différents systèmes de participation établis par les chambres préliminaires}

La première à établir un système de participation est la Chambre préliminaire I, le 17 janvier $2006^{6}$, à l'occasion des six premières demandes de participation reçues par la Cour de victimes dans la situation en RDC, décision dans laquelle le rôle des victimes dans la procédure était affirmé avec force:

De l'avis de la Chambre, le Statut confère aux victimes une voix et un rôle indépendants dans la procédure devant la Cour. Cette indépendance doit pouvoir s'exercer à l'égard du procureur de la Cour pénale internationale afin que les victimes puissent exprimer leurs intérêts. Ainsi que la Cour européenne l'a réitéré à plusieurs reprises, une victime qui participe à la procédure pénale ne peut être considérée comme l'adversaire du ministère public, ni d'ailleurs nécessairement comme son alliée, leur rôle et leurs objectifs étant clairement distincts?

La Chambre précisait en outre que l'article 68, paragraphe 3, du Statut donnait un droit d'accès général des victimes à la procédure devant la Cour, un droit d'autant plus crucial pendant l'enquête du procureur que, comme la Chambre le rappelait, c'était à ce stade que les affaires étaient sélectionnées. La participation des victimes à la procédure avait donc en partie un but répressif puisque l'article 68, paragraphe 3, du Statut conférait aux victimes le droit de participer à la lutte contre l'impunité 8 . Par ailleurs, dans le système établi par le Statut de la CPI, qui ne prévoit pas la possibilité d'une réparation pour les victimes sans des poursuites et une condamnation préalables, l'instance civile en réparation dépendant donc totalement de l'instance pénale, la participation des victimes au stade de l'enquête était d'autant plus importante que la poursuite était la condition sine qua non de l'accès aux réparations ${ }^{9}$ pour les victimes.

6. CPI, Situation en République démocratique du Congo, ICC-01/04-101, Chambre préliminaire I, Décision sur les demandes de participation à la procédure de VPRS 1 , VPRS 2 , VPRS 3, VPRS 4, VPRS 5 et VPRS 6, 17 janvier 2006.

7. Idem, paragraphe 51.

8. Ibidem, paragraphe 53 .

9. Ibidem, paragraphe 72 . 
La Chambre préliminaire I fixait de la manière suivante les modalités de participation des victimes de la situation pendant le déroulement de l'enquête:

a) présenter leurs vues et préoccupations à la Chambre et déposer des pièces en relation avec l'enquête en cours, nonobstant toute procédure spécifique ayant lieu dans le cadre d'une telle enquête ${ }^{10}$;

b) concernant des procédures spécifiques à l'enquête, trois cas de figure $^{11}$ étaient distingués en fonction de l'incidence de ces procédures sur leurs intérêts personnels: i) les procédures initiées par la Chambre de sa propre initiative auxquelles la Chambre pouvait inviter les victimes à participer; ii) les procédures initiées par d'autres participants (le procureur par exemple) pour lesquelles la Chambre déciderait si les victimes pouvaient y participer; et iii) les procédures initiées par les victimes elles-mêmes, la Chambre reconnaissant aux victimes le droit de lui demander d'ordonner des mesures spécifiques, sans préciser quelles pouvaient être ces mesures.

L'année suivante, en août 2007, la Chambre préliminaire II $^{12}$ établissait «son» système pour la participation des victimes de la situation à la procédure devant la Cour. Alors qu'elle indiquait sa volonté d'établir un système aboutissant à la certitude et l'efficacité de la participation des victimes de la situation à la procédure devant la Cour, la Chambre se contentait d'énumérer les dispositions du Statut pour l'application desquelles on pouvait envisager une telle participation: i) l'article 53 du Statut, et plus particulièrement son paragraphe 3 qui prévoit la possibilité pour la Chambre préliminaire de revoir les décisions du procureur de ne pas enquêter ou de ne pas poursuivre, était présenté comme le scénario le plus important lors duquel les victimes pouvaient jouer un rôle particulièrement influent ${ }^{13}$; et ii) les articles 56 et 57 , paragraphe $3 \mathrm{c}$ ), du Statut, qui visent notamment la possibilité pour la Chambre préliminaire de prendre des mesures de protection à l'égard des victimes, mais aussi de prendre des mesures visant à préserver des

10. Ibidem, paragraphe 71 .

11. Ibidem, paragraphes 73 à 75 .

12. CPI, Situation in Uganda, ICC-02/04-101, Pre-Trial Chamber II, Decision on victims' applications for participation a/0010/06, a/0064/06, to a/0070/06, a/0081/06 to a/0104/06 and a/0111/06 to a/0127/06 (uniquement disponible en anglais), 10 août 2007.

13. Idem, paragraphe 93. 
preuves $^{14}$, étaient également des articles pour l'application desquels les victimes pouvaient jouer un rôle. La Chambre rappelait enfin que la règle 93 du Règlement lui laissait la possibilité d'inviter les victimes de la situation à présenter leurs vues sur toute question qui lui paraissait appropriée ${ }^{15}$.

Si la Chambre préliminaire II admettait la participation des victimes de la situation à la procédure, comme l'avait fait avant elle la Chambre préliminaire I, le système institué était bien moins ouvert: en effet, il n'était pas question de laisser un droit général pour les victimes de présenter leurs vues et préoccupations ainsi que des pièces à la Chambre en relation avec l'enquête menée par le procureur et ce, nonobstant toute procédure spécifique. Par ailleurs, un droit d'initiative procédural des victimes n'était reconnu expressément par la Chambre préliminaire II qu'à l'égard des mesures de protection en faveur des victimes ${ }^{16}$, alors que la Chambre préliminaire I avait admis le droit pour les victimes de solliciter l'adoption de mesures par la Chambre préliminaire, sans spécifier à quoi pouvaient se rapporter ces mesures.

Ces deux systèmes allaient s'appliquer parallèlement dans la situation en Ouganda, dont la Chambre préliminaire II était saisie, ainsi que pour les situations en RDC et au Darfour, dont la Chambre préliminaire I était saisie, jusqu'à la décision de la Chambre d'appel rendue le 19 décembre $2008^{17}$.

Dans cet arrêt, la Chambre d'appel critique le droit pour les victimes de participer au stade de l'enquête en dehors de toute procédure judiciaire, et plus particulièrement le paragraphe 71 de la décision du 17 janvier 2006, alors même que cette décision ne faisait pas l'objet d'un appel, qui donnait le droit aux victimes de présenter leurs vues et préoccupations et de déposer des pièces en relation avec l'enquête en cours. Pour la Chambre d'appel, la participation des victimes doit se limiter aux procédures judiciaires devant une chambre, ce qui signifie

14. Ibidem, paragraphes 100 et 101.

15. Ibidem, paragraphe 102 .

16. Ibidem, paragraphes 98 et 99 .

17. CPI, Situation en République démocratique du Congo, ICC-01/04-556-tFRA, Chambre d'appel, Arrêt relatif à la participation des victimes au stade de l'enquête dans le cadre de l'appel interjeté par le Bureau du conseil public pour la Défense contre la décision rendue le 7 décembre 2007 par la Chambre préliminaire I et de l'appel interjeté par le Bureau du conseil public pour la Défense et le Procureur contre la décision rendue le 24 décembre 2007 par la Chambre préliminaire I, 19 décembre 2008. 
que les victimes ne peuvent exprimer leurs vues et préoccupations visà-vis du processus d'enquête lui-même.

La Chambre d'appel justifie sa position par la définition qu'elle donne, au paragraphe 45 de son arrêt, du terme "procédure», un terme qui selon la Chambre d'appel «signifie qu'une affaire est pendante devant une chambre». La Chambre d'appel procède ici par affirmation et ne donne aucune justification à la définition proposée du terme procédure: la démarche est encore une fois curieuse car le Statut utilise le terme procédure à de multiples reprises (plus de quarante fois aussi bien dans la version française que dans la version anglaise) et que ce terme vise toutes les phases de la procédure se déroulant devant les organes de la Cour, y compris la phase de l'enquête et même la phase avant l'enquête, à savoir la phase de l'examen préliminaire. Par ailleurs, la définition donnée par la Chambre d'appel ne semble pas correspondre aux droits de l'Homme internationalement reconnus puisque la Cour européenne ${ }^{18}$, par exemple, prend en considération la période de l'enquête menée par le procureur pour vérifier si la procédure dans son ensemble a été conduite dans un délai raisonnable.

Si la décision de la Chambre d'appel était décevante, en ce qu'elle ne définissait en rien les droits procéduraux des victimes, elle a eu cependant un effet paralysant, non seulement sur la participation des victimes de la situation à la procédure devant la Cour, mais aussi sur le traitement de leurs demandes de participation par les chambres préliminaires: en effet, en 2009 et 2010, on ne trouve aucune décision concernant les demandes de participation à la procédure des victimes dans les situations en RDC et au Darfour, d'où les critiques des ONG sur les demandes des victimes en souffrance devant la Chambre préliminaire I, notamment en $\mathrm{RDC}^{19}$. Dans la situation en Ouganda, la dernière décision concernant les demandes de participation des victimes à la procédure remonte au 10 mars $2009^{20}$.

18. Voir notamment Cour européenne des droits de l'homme, Affaire Selmouni c. France, requête $\mathrm{n}^{\circ}$ 25803/94, arrêt du 28 juillet 1999, paragraphes 108 à 118.

19. CPI, Conférence de révision du Statut de Rome, Kampala, 31 mai-11 juin 2010, $\mathrm{RC} / \mathrm{ST} / \mathrm{V} / \mathrm{INF} .4$, L'impact du système du Statut de Rome sur les victimes et communautés affectées, voir page 4, les critiques notamment rapportées par l'ONG REDRESS.

20. CPI, Situation in Uganda, ICC-02/04-180, Decision on victims' applications for participation a/0192/07 to a/0194/07, a/0196/07, a/0200/07, a/0204/07 a/0206/07, a/0209/07, a/0212/07, a/0216/07, a/0216/07, a/0217/07, a/0219/07 to a/0221/07, a/0228/07 to a/0230/07, a/0234/07, a/0235/07, a/0237/07, a/0324/07 and a/0326/07 under rule 89 (uniquement disponible en anglais), 10 mars 2009. 
C'est dans ce contexte que la Chambre préliminaire II, dans une composition différente de celle qui avait pris les décisions entre 2007 et 2009 dans la situation en Ouganda, décidait, le 3 novembre $2010^{21}$, de proposer un troisième système de participation à la procédure pour les victimes de la situation au Kenya cette fois-ci. Ce système a été étendu aux victimes de la situation en République centrafricaine quelques jours plus tard 22 .

Contrairement aux décisions précédentes de la Chambre préliminaire I en 2006 et de la Chambre préliminaire II en 2007 qui ont établi un système de participation à la procédure des victimes de la situation à l'occasion d'une décision prise sur des demandes de participation à la procédure présentées par des victimes, la décision prise dans la situation au Kenya est une «décision cadre» («framework decision» en anglais) qui vise à établir un système pour la participation des victimes à la procédure dans la situation au Kenya, indépendamment des affaires que le procureur pourra éventuellement sélectionner pour ses poursuites dans cette situation, système qui est établi sans qu'une demande de participation des victimes à la procédure ait été présentée à la Chambre.

La Chambre préliminaire rappelle tout d'abord les droits fondamentaux des victimes à un recours effectif et à avoir accès à la justice, puis insiste sur la jurisprudence de la Chambre d'appel du 19 décembre 2008 qui limite la participation des victimes aux procédures judiciaires, épinglant au passage la Chambre d'appel pour son absence totale de précision sur les procédures auxquelles les victimes pourraient participer dans le cadre de l'enquête dans une situation ${ }^{23}$.

La Chambre préliminaire opère ensuite une combinaison entre les systèmes établis par les Chambres préliminaires I et II, respectivement en 2006 et 2007: à la décision prise en août 2007 par la Chambre

21. CPI, Situation in the Republic of Kenya, ICC-01/09-24, Pre-Trial Chamber II, Decision on Victims' Participation in Proceedings Related to the Situation in The Republic of Kenya (uniquement disponible en anglais), 3 novembre 2010.

22. CPI, Situation in the Central African Republic, ICC-01/05-31, decision on Victims' Participation in Proceedings Related to the Situation in the Central African Republic (uniquement disponible en anglais), 11 novembre 2010.

23. CPI, Situation in the Republic of Kenya, ICC-01/09-24, Pre-Trial Chamber II, Decision on Victims' Participation in Proceedings Related to the Situation in The Republic of Kenya (uniquement disponible en anglais), 3 novembre 2010; voir le paragraphe $11 \mathrm{de}$ la décision qui se lit comme suit dans sa version anglaise: "In particular, the Appeals Chamber Judgement of 19 December 2008, which addressed the question of victims' participation in the context of the situation, fell short of any guidance as to the possible scenarios that could lead to such participation at the situation stage.» 
préliminaire II, elle emprunte ${ }^{24}$ son catalogue de dispositions du Statut pour l'application desquelles les victimes de la situation pourraient participer. La Chambre fait ainsi référence aux articles 53, 56 et 57, paragraphe 3 c) du Statut. Elle rappelle également que la règle $93 \mathrm{du}$ Règlement lui permet de solliciter les vues des victimes sur toute question.

À la décision de la Chambre préliminaire I prise en 2006, elle emprunte ${ }^{25}$ les trois cas de figure mentionnés aux paragraphes 73 à 75 de cette décision, à savoir la possibilité d'une saisine par les victimes ${ }^{26}$, la possibilité pour la Chambre d'agir de sa propre initiative et la possibilité d'être saisie par un participant autre que les victimes. La Chambre préliminaire évite en revanche soigneusement de faire référence au paragraphe 71 de la décision prise le 17 janvier 2006 par la Chambre préliminaire I qui permettait aux victimes de présenter leurs vues et préoccupations nonobstant toute procédure spécifique, puisque ce paragraphe avait fait l'objet d'une censure par la Chambre d'appel.

Le paragraphe essentiel de la décision est le paragraphe 16, où la Chambre préliminaire détermine la façon dont elle va traiter les demandes de participation des victimes de la situation, ce qu'elle appelle le «cadre procédural» de leur «éventuelle» participation.

En effet, après la décision de la Chambre d'appel du 19 décembre 2008, deux options semblaient possibles: soit les chambres préliminaires recevaient de manière régulière les demandes de participation des victimes et décidaient si elles étaient victimes au regard de la règle 85 du Règlement, laissant à plus tard la détermination de leurs droits procéduraux en fonction des procédures concrètes qui pourraient se dérouler au stade de l'enquête dans la situation en cause; soit les chambres préliminaires renvoyaient l'examen au regard de la règle $85 \mathrm{du}$ Règlement au moment où les victimes pourraient éventuellement participer à des procédures se déroulant au stade de l'enquête, cette seconde option ayant le clair désavantage de bloquer toute participation des victimes pendant le temps nécessaire à les reconnaître en tant que victimes au regard de la règle 85 du Règlement avec le risque soit d'une

24. Idem, paragraphe 12 .

25. Ibidem, paragraphe 15 .

26. C'est peut-être le seul point positif de cette décision qui ouvre ainsi une petite porte aux victimes de la situation qui ont au moins le droit de saisir la Chambre préliminaire d'une requête. Il n'est bien entendu pas garanti que la suite donnée à cette requête soit positive. 
exclusion des victimes de ces procédures, soit d'un retard considérable dans le déroulement de ces procédures.

C'est malheureusement cette seconde option qui a été choisie par la Chambre préliminaire II dans la situation au Kenya puisqu'au paragraphe 16 de sa décision, elle indique clairement qu'elle ne procédera à l'examen des demandes de participation des victimes de la situation au Kenya que si elle a au préalable déterminé qu'une procédure judiciaire devait être conduite, soit qu'elle ait décidé d'initier elle-même une procédure comme elle le peut notamment en application des articles 53, paragraphe $3 \mathrm{~b}$ ), 56, paragraphe 3 , et 57 paragraphe $3 \mathrm{c}$ ), du Statut, soit qu'elle ait estimé que la requête présentée par les victimes ou d'autres participants pouvait effectivement conduire à une procédure judiciaire devant elle.

Il est donc clair que les demandes de participation des victimes dans la situation au Kenya pourront rester des années en souffrance au greffe de la Cour, dans l'attente qu'une procédure judiciaire puisse éventuellement se dérouler. Les victimes n'auront donc même plus de décision portant sur leur reconnaissance en tant que victime au regard de la règle $85 \mathrm{du}$ Règlement sauf si une procédure intervient en rapport avec l'enquête au Kenya. On peut se demander si une telle jurisprudence est conforme à la règle 89 du Règlement qui fait obligation au greffier de transmettre les demandes de participation des victimes à la Chambre compétente: ici, c'est la Chambre qui ordonne au greffier de garder ces demandes tant qu'une procédure n'intervient pas dans le cadre de l'enquête dans la situation, sans que l'on sache quand une telle procédure peut intervenir.

\section{Des droits procéduraux très théoriques}

En effet, les requêtes présentées par les victimes de la situation ont toutes été rejetées jusqu'à ce jour, ce qui amène à se poser la question de l'utilité d'établir des systèmes de participation à la procédure des victimes de la situation si en fait les requêtes de celles-ci n'aboutissent à rien, quels que soient les systèmes établis et la force des déclarations employées par les chambres préliminaires pour établir les droits des victimes, en faisant notamment référence à leur «voix» et leur «rôle» indépendants dans la procédure.

Ainsi, les victimes VPRS 1 à 6, reconnues en janvier 2006 par la Chambre préliminaire I, ont saisi en 2006, puis en 2010, la Chambre 
préliminaire I dans le but essentiellement de contester des décisions du procureur de ne pas poursuivre certains crimes. Ces victimes cherchaient également à obtenir, d'une part, des informations sur le statut de l'enquête en RDC en ce qui concerne les crimes dont elles avaient été victimes et, d'autre part, que la Chambre prenne des mesures aux fins de préserver des preuves.

En juin 2006, le procureur annonçait en effet qu'il suspendait l'enquête à l'égard d'autres charges qui pourraient être retenues contre Thomas Lubanga Dyilo: en conséquence, il renonçait à solliciter un amendement des charges pour en ajouter d'autres relatives notamment à des crimes de nature sexuelle. Le 30 août 2006, le représentant légal des victimes VPRS 1,4 et 5 demandait notamment à la Chambre préliminaire I d'examiner en vertu de l'article 53, paragraphe $3 \mathrm{~b}$ ), du Statut la décision implicite du procureur de ne pas poursuivre et de prendre les mesures nécessaires aux fins de préservation d'éléments de preuve.

Le 4 septembre 2006, le représentant légal des victimes VPRS 2, VPRS 3 et VPRS 6 sollicitait de la Chambre préliminaire I qu'elle demande au procureur de lui indiquer l'état de son enquête notamment sur les crimes commis contre VPRS 2, VPRS 3 et VPRS 6 et qu'elle prenne les mesures nécessaires afin de préserver des éléments de preuve.

La Chambre préliminaire $\mathrm{I}^{27}$ rejetait un an plus tard l'ensemble des requêtes présentées par les victimes VPRS 1 à VPRS 6 dans une décision assez laconique.

En effet, la Chambre se contente d'affirmer que le procureur n'a pas pris de décision de ne pas poursuivre puisqu'il a seulement «suspendu» son enquête dans la situation en RDC, en raison de la situation précaire en matière de sécurité et, qu'en conséquence, à défaut de décision prise par le procureur, la demande du représentant légal visant à ce que la Chambre préliminaire utilise ses pouvoirs sous l'angle de l'article 53, paragraphe 3 b) du Statut pour revoir cette décision n'est pas fondée en droit.

La motivation laisse perplexe lorsque l'on sait que le procureur avait affirmé avoir renoncé à présenter des charges supplémentaires contre Thomas Lubanga Dyilo, ce qui suppose qu'il avait pris une décision de

27. CPI, Situation en République démocratique du Congo, ICC-01/04-373-tFRA, Chambre préliminaire I, Décision sur les demandes du représentant légal des victimes VPRS 1 à VPRS 6 relatives aux informations fournies par le procureur concernant la poursuite de l'enquête, 26 septembre 2007. 
ne pas le poursuivre pour ces crimes. Cette suspension temporaire de l'enquête n'avait aucune base légale dans le Statut et devait forcément s'analyser comme une décision de ne pas poursuivre.

Cette décision présentée comme temporaire en juin 2006, apparaissait au surplus de plus en plus définitive un an plus tard, quand la Chambre statuait en septembre 2007, d'autant plus que les charges présentées initialement contre Thomas Lubanga Dyilo avaient été confirmées le 29 janvier $2007^{28}$ par la Chambre préliminaire I et qu'une chambre de première instance avait été constituée pour son jugement pour ces crimes ${ }^{29}$. On attend toujours d'ailleurs, en 2011, la présentation par le procureur de charges supplémentaires contre Thomas Lubanga Dyilo: ceci ne serait plus possible dans le cadre de l'affaire dont est actuellement saisie la Chambre de première instance I puisque l'article 61, paragraphe 9, du Statut interdit la modification des charges après le début du procès, si ce n'est pour supprimer des charges.

Sur ce point il est donc intéressant de constater que la Chambre préliminaire I s'est réfugiée derrière l'absence de décision formelle et notifiée à la Chambre préliminaire par le procureur de ne pas poursuivre, afin de justifier son inaction. Ce faisant, elle offre au procureur une tactique très simple pour éviter tout contrôle judiciaire sur ses décisions de ne pas poursuivre: il lui suffit de ne pas matérialiser ses décisions et donc de ne pas les notifier à la Chambre préliminaire. Cette dernière n'a même pas fait l'effort de solliciter des renseignements du procureur sur ses intentions en matière de poursuites ou même sur le délai prévisible de cette suspension «temporaire».

En ce qui concerne la requête tendant à la préservation des preuves, la Chambre se contentait d'affirmer qu'elle n'en voyait pas le besoin car rien n'indiquait que le procureur n'avait pas pris les mesures nécessaires pour assurer la préservation des preuves. Là encore, la Chambre ne demandait aucun renseignement précis au procureur sur ce point.

S'agissant enfin de la requête visant à obtenir des renseignements sur l'état de l'enquête du procureur au regard des crimes commis contre les victimes VPRS 2, 3 et 6, la Chambre n'y répondait tout simplement pas.

28. CPI, Situation en République démocratique du Congo, Affaire le Procureur c. Thomas Lubanga Dyilo, ICC-01/04-01/06-803, Chambre préliminaire I, Décision sur la confirmation des charges, 29 janvier 2007.

29. CPI, Situation en République démocratique du Congo, Affaire le Procureur c. Thomas Lubanga Dyilo, ICC-01/04-01/06-841-tFRA, Présidence, Décision portant constitution de la Chambre de première instance I et renvoi à celle-ci de l'affaire Le Procureur $c$. Thomas Lubanga Dyilo, 6 mars 2007. 
Pourtant le paragraphe 6 a) de l'Annexe à la Déclaration des principes fondamentaux de justice relatifs aux victimes de criminalité et aux victimes d'abus de pouvoir, Déclaration adoptée le 29 novembre 1985 par l'Assemblée générale des Nations Unies ${ }^{30}$ à laquelle la Chambre préliminaire $\mathrm{I}^{31}$ ainsi que la Chambre préliminaire III $^{32}$ s'étaient référées dans leur jurisprudence, précise que les victimes doivent être informées des dates et du déroulement des procédures et de l'issue de leurs affaires, spécialement lorsqu'il s'agit d'actes criminels graves et lorsqu'elles ont demandé ces informations. En l'espèce, il s'agissait de crimes très graves et les victimes avaient demandé ces informations.

$\mathrm{Si}$, par ailleurs, comme l'affirme la Chambre d'appel ${ }^{33}$, «les droits de l'homme sous-tendent le Statut dans tous ses aspects», alors il convient de rappeler que la Cour européenne des droits de l'homme a rendu de nombreuses décisions affirmant le droit des victimes à être informées des enquêtes portant sur les crimes dont elles ont souffert. Ces informations doivent porter non seulement sur l'ouverture de l'enquête mais aussi sur son déroulement ${ }^{34}$.

Cette première décision à la suite d'une requête des victimes de la situation n'était guère encourageante: cependant, le 28 juin 2010, le représentant légal des victimes VPRS 3 et 6 déposait une nouvelle requête ${ }^{35}$ devant la Chambre préliminaire I tendant à voir Jean-Pierre

30. Résolution 40/34, document des Nations Unies, A/RES/40/34.

31. CPI, Situation en République démocratique du Congo, ICC-01/04-101, Chambre préliminaire I, Décision sur les demandes de participation à la procédure de VPRS 1 , VPRS 2, VPRS 3, VPRS 4, VPRS 5 et VPRS 6, 17 janvier 2006, paragraphe 115.

32. CPI, Situation en République centrafricaine, Affaire le Procureur c. Jean-Pierre Bemba Gombo, ICC-01/05-01/08-320-tFRA, Chambre préliminaire III, Quatrième décision relative à la participation des victimes, 12 décembre 2008, paragraphe 16 .

33. CPI, Situation en République démocratique du Congo, Affaire le Procureur c. Thomas Lubanga Dyilo, ICC-01/04-01/06-772-tFRA, Chambre d'appel, Arrêt relatif à l'appel interjeté par Thomas Lubanga Dyilo contre la décision du 3 octobre 2006 relative à l'exception d'incompétence de la Cour soulevée par la Défense en vertu de l'article 19-2-a du Statut, 14 décembre 2006, paragraphe 37.

34. Cour européenne des droits de l'homme, Affaire Gülec c. Turquie, 54/1997/838/1044, 27 août 1998, paragraphe 82; Affaire Ogur c. Turquie, requête nº 21594/93, 20 mai 1999, paragraphe 92; Case of Kelly and others v. United Kingdom (uniquement disponible en anglais), requête $n^{\circ}$ 30054/96, 4 mai 2001, paragraphe 98; Case of Alikhadzbiyeva v. Russia (uniquement disponible en anglais), requête $\mathrm{n}^{\circ}$ 68007/01, 5 juillet 2007, paragraphe 72 .

35. CPI, Situation en République démocratique du Congo, ICC-01/04-564, représentant légal des victimes VPRS 3 et 6 , Demande du représentant légal de VPRS 3 et 6 aux fins de mise en cause de Monsieur Jean-Pierre Bemba en sa qualité de chef militaire au sens de l'article 28-a du Statut pour les crimes dont ses troupes sont présumées coupables en Ituri, 28 juin 2010. 
Bemba Gombo poursuivi non seulement pour les crimes prétendument commis par ses troupes en République centrafricaine, mais aussi pour ceux prétendument commis en Ituri en octobre 2002, crimes pour lesquels le procureur n'avait exercé aucune poursuite alors qu'il affirmait détenir des éléments de preuve sur les crimes commis par les troupes de Jean-Pierre Bemba Gombo lors de leur offensive en Ituri à cette date.

Pour prouver que le procureur avait pris en réalité une décision de ne pas poursuivre Jean-Pierre Bemba Gombo pour les crimes prétendument commis par ses troupes en Ituri en octobre 2002, le représentant légal, dans un mémoire long de 16 pages, reprenait toutes les déclarations publiques du procureur dans lesquelles il affirmait très clairement, y compris dans un rapport officiel de la CPI aux Nations Unies en 2008, que ses enquêtes sur les crimes commis en Ituri étaient à présent terminées.

Dans une décision rendue le 25 octobre $2010^{36}$, la Chambre préliminaire I a rejeté la requête du représentant légal. Il est intéressant de noter que la Chambre préliminaire n'a en rien examiné les faits présentés dans la requête mais s'est contentée de rappeler que le procureur avait déclaré à la Chambre qu'il n'avait pas pris de décision de ne pas poursuivre Jean-Pierre Bemba Gombo pour les crimes prétendument commis en Ituri et dès lors, compte tenu des informations en sa possession, elle ne voyait aucune raison de ne pas croire le procureur. La motivation tient en deux paragraphes de trois lignes chacun. C'est une confirmation de la jurisprudence du 26 septembre 2007 : il suffit pour le procureur de dire qu'il n'a pris aucune décision pour que la Chambre se retranche derrière les déclarations du procureur et refuse d'exercer tout contrôle. La Chambre n'exige même pas du procureur qu'il conduise ses enquêtes, comme l'exige la jurisprudence de la Cour européenne des droits de l'homme ${ }^{37}$, dans un délai raisonnable: en l'espèce les faits remontaient à 2002 et l'ouverture de l'enquête en RDC à 2004.

Une telle jurisprudence revient à laisser au procureur le choix à la fois de l'éventualité de l'exercice du contrôle judiciaire et le choix du

36. CPI, Situation in the Democratic Republic of the Congo, ICC-01/04-582, Pre-Trial Chamber I, Decision on the request of the legal representative of victims VPRS 3 and VPRS 6 to review an alleged decision of the Prosecutor not to proceed (uniquement disponible en anglais), 25 octobre 2010.

37. Cour européenne des droits de l'homme, Case of Bazorkina v. Russia (uniquement disponible en anglais), requête $\mathrm{n}^{\circ} 69481 / 01,27$ juillet 2006, paragraphe 119. 
moment de ce contrôle puisque la Chambre préliminaire ne lui fixe aucune limite de temps pour prendre sa décision et la notifier. Autrement dit, si la Chambre préliminaire vient à exercer ce contrôle judiciaire sur les décisions du procureur de ne pas poursuivre, c'est parce que celui-ci souhaite qu'elle exerce un tel contrôle, ce qui en réduit singulièrement la portée et l'efficacité.

Les juges, sacrifiant au passage l'intérêt légitime des victimes, ont sans doute voulu d'abord laisser une totale liberté d'action au procureur et ensuite éviter ainsi de prendre la moindre part de responsabilité dans sa politique de sélection des affaires. D'autres décisions des Chambres préliminaires $\mathrm{I}^{38}$ et $\mathrm{II}^{39}$ confirment cette tendance qui semble peu se soucier des droits de l'homme internationalement reconnus ${ }^{40}$.

Les organisations de défense des droits de l'homme, dont la FIDH, ont protesté, à la suite de cette décision du 25 octobre 2010 de la Chambre préliminaire I, par un communiqué du 3 novembre $2010^{41}$ qui rappelle la raison d'être de la Chambre préliminaire:

Nos organisations trouvent problématique que la Chambre préliminaire refuse dans les faits d'assumer son rôle de contrôle des décisions et omissions du Procureur dans la phase préliminaire de la procédure. La création de cette chambre avait précisément pour objectif, lors de l'adoption du

38. CPI, Situation en République démocratique du Congo, ICC-01/04-373-tFRA, Chambre préliminaire $\mathrm{I}$, Décision relative à la demande présentée en vertu de la règle 103-1 du Règlement de procédure et de preuve, 17 août 2007.

39. CPI, Situation in the Central African Republic, Case of the Prosecutor v. Jean-Pierre Bemba Gombo, ICC-01/05-01/08-453, Pre-Trial Chamber II, Decision on Request for Leave to Submit Amicus Curiae Observations Pursuant to Rule 103 of the Rules of Procedure and Evidence (uniquement disponible en anglais), 17 juillet 2009, paragraphe 10.

40. Voir par exemple la Recommandation Rec (2000)19 du Comité des Ministres aux États membres sur le rôle du ministère public dans le système de justice pénale adoptée par le Comité des Ministres du Conseil de l'Europe le 6 octobre 2000; le paragraphe 34 de cette recommandation est ainsi rédigé:

Les parties intéressées à l'affaire, lorsqu'elles sont reconnues comme telles ou identifiables, en particulier les victimes, doivent avoir la possibilité de contester la décision prise par le ministère public de ne pas engager de poursuites; une telle contestation peut se faire, le cas échéant après contrôle hiérarchique, soit dans le cadre d'un contrôle juridictionnel, soit en autorisant les parties à mettre en œuvre elles-mêmes les poursuites.

Dans le même sens et faisant référence à cette Recommandation, voir Cour européenne des droits de l'homme, Affaire Perez contre France, 12 février 2004, Grande Chambre, requête $\mathrm{n}^{\circ} 47287 / 99$, paragraphe 68 .

41. FIDH, RDC/CPI, 3 novembre 2010: «Les victimes interrogent la CPI sur l'absence de poursuites contre Jean-Pierre Bemba pour des crimes commis en RDC - Les juges les déboutent déclarant que l'enquête du Procureur sur la RDC est toujours ouverte», disponible sur le site internet de la FIDH, www.fidh.org. 
Statut de Rome, de mettre en place un certain système d'évaluation des décisions du Procureur.

La jurisprudence de la CPI sur les droits procéduraux des victimes de la situation doit sans aucun doute leur laisser un goût amer: elles n'ont pour l'instant aucune manière d'influer sur les choix du procureur ni même la possibilité de les discuter devant les juges. L'information reçue sur ces choix et leur justification est quasiment inexistante. Il faudrait sur ce point une véritable modification de la jurisprudence de la CPI pour aboutir à un droit pour les victimes de déclencher un mécanisme de contrôle judiciaire («judicial review» en anglais) des décisions du procureur de ne pas enquêter ou de ne pas poursuivre certaines affaires, après avoir reçu une information claire et détaillée sur les choix effectués par le procureur et les raisons de ces choix. Ceci obligerait le procureur à mieux justifier ses choix et contribuerait à ne pas laisser un sentiment d'arbitraire aux victimes qui demeurent en dehors de ses choix. Il est possible en droit et souhaitable pour l'intérêt des victimes et la crédibilité de l'institution qu'une telle possibilité soit ouverte aux victimes, conformément aux droits de l'homme internationalement reconnus. Les victimes auraient ainsi une réelle possibilité de pouvoir participer à la lutte contre l'impunité particulièrement dans les cas où le procureur se refuse à enquêter ou à poursuivre pour des motifs de pure opportunité. Il ne faut enfin pas oublier que dans le système institué par le Statut de la CPI, le droit des victimes à solliciter réparation des préjudices subis dépend de l'ouverture d'une enquête et de poursuites: les victimes ont donc un intérêt particulier à pouvoir contester les choix du procureur.

\section{Les droits procéduraux des victimes de l'affaire}

Le rôle des victimes après la sélection d'une affaire par le procureur diffère en fonction du stade de la procédure: si leur rôle durant la phase préliminaire est particulièrement limité (1), on note une certaine avancée au stade du procès (2).

\section{Le rôle des victimes au stade préliminaire de l'affaire}

La phase préliminaire d'une affaire se déroule de la requête du procureur aux fins de délivrance par la Chambre préliminaire d'un mandat d'arrêt ou d'une citation à comparaître en application de l'article 58 du Statut, 
jusqu'à la décision de la Chambre préliminaire sur la confirmation des charges en application de l'article 61, paragraphe 7, du Statut, décision par laquelle la Chambre préliminaire décide du renvoi ou non de la personne poursuivie devant une chambre de première instance pour être jugée. Cette phase de la procédure se déroule en général sur une période d'une année environ.

Là encore chaque chambre préliminaire a tenté de définir son «système de participation» des victimes lors de la phase préliminaire de l'affaire, système qui varie d'une chambre à l'autre, voire au sein d'une même chambre en fonction des affaires, pour aboutir le plus souvent à une participation largement symbolique des victimes à la procédure, alors même que la phase est cruciale puisqu'elle détermine le cadre factuel du procès: en effet, en application de l'article 61, paragraphe 7 a) du Statut, en cas de confirmation des charges par la Chambre préliminaire, la personne est renvoyée devant la Chambre de première instance pour y être jugée exclusivement sur la base des charges ainsi confirmées. Si le procureur souhaite une modification des charges ainsi confirmées, il doit en demander l'autorisation à la Chambre préliminaire.

C'est la Chambre préliminaire I, à la suite de la première remise à la CPI d'une personne poursuivie, à savoir Thomas Lubanga Dyilo, qui a statué en premier sur la question des droits procéduraux des victimes à ce stade de la procédure ${ }^{42}$, se limitant cependant à leur participation à l'audience sur la confirmation des charges.

Il était en effet particulièrement important de fixer tout d'abord les limites et le but de l'intervention des victimes dans le cadre de l'audience de confirmation des charges. Sur ce point, le passage pertinent de la décision du 22 septembre 2006 est ainsi rédigé:

Attendu en conséquence que, sous réserve que leur intervention se limite au cadre fixé par les charges portées à l'encontre de Thomas Lubanga Dyilo, les victimes peuvent participer à cette audience de confirmation des charges en exposant leurs vues et préoccupations afin de contribuer utilement à la répression des crimes dont elles allèguent avoir souffert et de pouvoir, le cas échéant, obtenir ultérieurement réparation des préjudices subis.

42. CPI, Situation en République démocratique du Congo, Affaire le Procureur c. Thomas Lubanga Dyilo, ICC-01/04-01/06-462, Chambre préliminaire I, Décision sur les modalités de participation des victimes a/001/06, a/002/06 et a/003/06 à l'audience de confirmation des charges, 22 septembre 2006. 
La limite de l'intervention des victimes est ici clairement fixée: il s'agit des charges présentées par le procureur. Les victimes ne peuvent, dans le cadre de l'audience de confirmation des charges, tenter d'élargir les charges présentées par le procureur. On constate une certaine cohérence dans l'action des juges, qui après avoir rejeté toute possibilité pour les victimes de la situation de contester la sélection des affaires opérée par le procureur, veulent bloquer toute velléité des victimes de l'affaire de contester la sélection des charges opérée par le procureur dans une affaire donnée. On voit mal dès lors la traduction concrète de l'affirmation faite dans la décision du 17 janvier 2006 selon laquelle ${ }^{43}$ l'article 68 , paragraphe 3, du Statut confère «aux victimes le droit de participer à la lutte contre l'impunité».

Le but de la participation des victimes à l'audience de confirmation des charges est également clairement fixé: il s'agit de contribuer utilement à la répression des crimes dont elles allèguent avoir souffert. Il convient alors de se demander quels sont les moyens procéduraux dont disposent les victimes pour contribuer utilement à la répression de ces crimes et pouvoir ultérieurement obtenir réparation des préjudices subis.

En l'espèce l'affaire Thomas Lubanga Dyilo se distinguait par le fait que toutes les victimes qui avaient demandé à participer à la procédure avaient également demandé à conserver un anonymat total à l'égard de la personne poursuivie. Eu égard à cette exigence essentielle pour les victimes dans cette affaire, la Chambre préliminaire fixait des modalités de participation à la procédure qui lui paraissaient compatibles avec l'anonymat de ces victimes. Le passage pertinent de la décision du 22 septembre 2006 est ainsi rédigé :

Attendu qu'en principe, la participation anonyme des dites victimes à ce stade de la procédure devrait se limiter à i) un accès aux documents publics uniquement et ii) une présence aux audiences publiques uniquement; mais que la Chambre se réserve la possibilité de faire une exception à ce principe en cas de circonstances exceptionnelles,

Attendu qu'en vertu de la règle 89-1 du Règlement, les victimes ont la possibilité de faire des déclarations au début et à la fin des audiences auxquelles elles sont conviées,

Attendu que le représentant légal des victimes a la possibilité de demander l'autorisation d'intervenir lors des sessions publiques de l'audience de

43. CPI, Situation en République démocratique du Congo, ICC-01/04-101, Chambre préliminaire I, Décision sur les demandes de participation à la procédure de VPRS 1, VPRS 2, VPRS 3, VPRS 4, VPRS 5 et VPRS 6, 17 janvier 2006, paragraphe 53. 
confirmation des charges et que la Chambre se prononcera au cas par cas sur la base des principes établis dans la présente décision,

Attendu cependant qu'il serait porté atteinte au principe fondamental interdisant les accusations anonymes s'il était permis aux victimes a/001/06 à a/003/06 d'ajouter quelque élément de fait ou de preuve que ce soit au dossier présenté à l'encontre de Thomas Lubanga Dyilo par l'Accusation dans le document de notification des charges et l'inventaire des éléments de preuve; et que de l'avis de la Chambre, il s'ensuit que les victimes a/001/06 à a/003/06 ne sauraient interroger les témoins selon la procédure prévue à la règle 91-3 du Règlement.

Les moyens procéduraux dont disposaient les victimes dans l'affaire Thomas Lubanga Dyilo étaient donc singulièrement limités du fait de l'anonymat que celles-ci avaient requis, puisque les victimes n'avaient pas la possibilité d'ajouter des éléments de preuve ou de fait à ceux du dossier présenté par le procureur.

Par ailleurs, les victimes n'avaient accès ni aux documents confidentiels, ni aux éléments de preuve présentés par le procureur ou la défense. On peut légitimement se poser la question suivante: comment réellement participer à un débat judiciaire sans connaitre les éléments de preuve versés au débat ni pouvoir en apporter de nouveaux? Il faut reconnaitre qu'une telle participation des victimes se limite en fait essentiellement à la présence de leurs représentants légaux dans la salle d'audience: c'est une participation «de façade». Ceci est d'autant plus curieux que la règle 121-10 du Règlement précise qu'il est établi un dossier dans lequel sont versés les éléments de preuve présentés par le procureur et la défense, et que ce dossier peut être consulté par le procureur, la personne poursuivie ainsi que les victimes ou leurs représentants légaux, sans que le texte indique le moins du monde que les victimes ou leurs représentants légaux devraient avoir un accès moindre à ce dossier que le procureur ou la défense.

La motivation retenue par la Chambre pouvait cependant laisser penser que si les victimes ou au moins certaines d'entre elles n'avaient pas requis l'anonymat, elles auraient été autorisées à présenter des éléments de preuve supplémentaires à ceux présentés par le procureur. La solution dégagée par la Chambre n'en mettait pas moins les victimes dans une position délicate, à savoir soit bénéficier d'une participation à la procédure très réduite, soit risquer de se mettre en danger.

La Chambre préliminaire I se penchait à nouveau sur la distinction entre victimes anonymes et victimes non anonymes au regard de leurs 
droits procéduraux dans une décision rendue le 13 mai $2008^{44}$ dans l'affaire Germain Katanga et Mathieu Ngudjolo Chui. En effet, dans cette affaire, certaines victimes demandaient à ce que leur identité ne soit pas communiquée aux personnes poursuivies, alors que d'autres acceptaient qu'elle le soit. En revanche, toutes les victimes participant à la procédure demandaient et obtenaient de la Chambre que leur identité ne soit communiquée ni au public, ni aux médias. La Chambre préliminaire ordonnait aux équipes de la défense des deux personnes poursuivies dans cette affaire de ne désigner les victimes que par les numéros qui leur avaient été attribués par le Greffe.

S'agissant des droits procéduraux spécifiques des victimes non anonymes, la Chambre les divisait en six groupes ${ }^{45}$. Le premier groupe se rapportait au droit d'avoir généralement accès, avant et pendant l'audience de confirmation des charges, au dossier de l'affaire conservé par le Greffe, y compris aux éléments de preuve déposés par le procureur ou la défense en application de la règle 121 du Règlement. Ce droit englobait le droit de consulter l'ensemble des documents et décisions contenus dans le dossier de l'affaire, qu'ils soient publics ou confidentiels.

Le deuxième groupe comprenait le droit de présenter des conclusions sur toutes les questions relatives à l'admissibilité et à la valeur probante des éléments de preuve sur lesquels le procureur ou la défense entendaient se fonder à l'audience de confirmation des charges ou d'examiner ces éléments de preuve lors de cette audience.

Le troisième groupe comprenait, conformément à la règle $91 \mathrm{du}$ Règlement, le droit d'interroger les témoins comparaissant à l'audience. La Chambre pouvait cependant donner des instructions quant à ces interrogatoires menés par les représentants légaux des victimes, et le procureur comme la défense pouvaient demander à la Chambre, une fois la question posée et avant que le témoin n'y réponde, de ne pas admettre ladite question ou de demander au représentant légal de la victime de la reformuler.

Le quatrième groupe comprenait le droit d'assister à toutes les audiences publiques ou à huis clos convoquées dans le cadre des travaux menant à l'audience de confirmation des charges, ainsi qu'à toutes les

44. CPI, Situation en République démocratique du Congo, Affaire le Procureur c. Germain Katanga et Matbieu Ngudjolo Chui, ICC-01/04-01/07-474-tFRA, Chambre préliminaire I, Décision relative à l'ensemble des droits procéduraux associés à la qualité de victime dans le cadre de la procédure préliminaire en l'espèce, 13 mai 2008.

45. Ibidem, paragraphes 127 à 143. 
séances publiques et à huis clos de l'audience de confirmation des charges.

Le cinquième groupe comprenait le droit de participer à la procédure en présentant oralement des requêtes, réponses et conclusions et enfin, le sixième groupe comprenait le droit de présenter ces requêtes, réponses et conclusions par écrit.

En revanche, la Chambre refusait d'accorder aux victimes, même non anonymes, le droit de présenter des éléments de preuve supplémentaires par rapport à ceux présentés par le procureur ou la défense, contrairement à ce que laissait supposer sa décision du 22 septembre 2006 prise dans l'affaire Thomas Lubanga Dyilo. Pour ce faire, la Chambre affirmait que l'article 69, paragraphe 3, du Statut, qui permet à la Cour de demander la présentation de tous les éléments de preuve qu'elle juge nécessaires à la manifestation de la vérité, n'était applicable que devant la Chambre de première instance. Pourtant, l'article 69, paragraphe 3, fait référence à la «Cour», et pas seulement à la Chambre de première instance, contrairement à l'article $74 \mathrm{du}$ Statut par exemple. La règle 122(9) du Règlement, qui concerne l'audience de confirmation des charges, précise d'ailleurs que l'article 69 est applicable à cette audience.

Les droits accordés aux victimes anonymes étaient eux beaucoup plus restreints, suivant en cela la jurisprudence établie dans l'affaire Thomas Lubanga Dyilo, puisque la Chambre, encore une fois, rappelait le «principe fondamental interdisant les accusations anonymes». Cette affirmation est difficile à comprendre puisque les victimes n'ont de toute façon pas la capacité de présenter des éléments de preuve supplémentaires et on comprend donc mal de quelles accusations il est question.

Malgré ses limitations en ce qui concerne la présentation d'éléments de preuve supplémentaires par rapport à ceux présentés par le procureur ou la défense, cette décision du 13 mai 2008 reste à ce jour la décision la plus généreuse prise à l'égard de la participation des victimes lors de la phase préliminaire d'une affaire. C'est notamment la seule décision qui a permis aux représentants légaux des victimes d'avoir accès à tous les documents, qu'ils soient publics ou confidentiels, contenus au dossier de la procédure ainsi qu'à tous les éléments de preuve présentés par le procureur ou la défense, y compris aux procès-verbaux des interrogatoires menés par le procureur, quel que soit leur niveau de confidentialité. C'est donc la décision à ce jour qui a le mieux permis aux représentants légaux des victimes d'avoir une réelle possibilité de participer utilement au débat judiciaire devant la Chambre préliminaire. 
Cette jurisprudence n'a en effet été suivie ni par la Chambre préliminaire III dans l'affaire Jean-Pierre Bemba Gombo, ni par la Chambre préliminaire I elle-même, dans les autres affaires dont elle a été saisie dans la situation au Darfour.

En effet, dans sa décision relative à la participation des victimes dans l'affaire Jean-Pierre Bemba Gombo ${ }^{46}$, la Chambre préliminaire III décidait que les représentants légaux des victimes pourraient seulement avoir accès aux audiences publiques, aux décisions et documents publics ainsi qu'aux éléments de preuve publics.

Cependant, le procureur avait décidé de présenter tous les procèsverbaux des témoignages à la Chambre préliminaire comme éléments de preuve confidentiels et aucun témoin n'était appelé à comparaître devant la Chambre préliminaire lors de l'audience de confirmation des charges, ce qui aboutissait à laisser largement les représentants légaux des victimes en dehors du débat judiciaire, alors même que ceux-ci pouvaient utilement y contribuer eu égard à leur connaissance de la situation en République centrafricaine ${ }^{47}$.

De manière plus intéressante encore, la Chambre préliminaire I décidait de ne pas suivre sa propre jurisprudence dans les affaires émanant de la situation au Darfour. En effet, dans l'affaire Abu Garda ${ }^{48}$, la Chambre affirmait que l'article 68, paragraphe 3, du Statut, ainsi que les règles 91 et 92 du Règlement, lui permettaient de limiter la participation des victimes à la procédure soit de sa propre initiative, soit à la requête du procureur, de la défense mais aussi à la requête du greffe ou de tout autre participant à la procédure, s'il était démontré que ces limitations étaient nécessaires pour sauvegarder un autre intérêt tel que l'enquête du procureur, la sécurité nationale ou le bien-être physique ou psychologique des victimes ou des témoins ${ }^{49}$. L'affirmation laisse

46. Idem, paragraphes 101 à 110 .

47. CPI, Situation en République centrafricaine, Affaire le Procureur c. Jean-Pierre Bemba Gombo, ICC-01/05-01/08-424-tFRA, Chambre préliminaire III, Décision rendue en application des alinéas a) et b) de l'article 61-7 du Statut de Rome, relativement aux charges portées par le Procureur à l'encontre de Jean-Pierre Bemba Gombo, 15 juin 2009, paragraphe 106. Ce paragraphe est particulièrement intéressant puisque la Chambre fait référence à une intervention d'un représentant légal des victimes pendant l'audience pour l'identification des troupes suspectées d'avoir commis des crimes contre la population civile en République centrafricaine.

48. CPI, Situation in Darfur, Sudan, In the Case of the Prosecutor v. Babar Idriss Abu Garda, ICC-2/05-02/09-136, Pre-Trial Chamber I, Decision on victims' modalities of participation at the Pre-Trial Stage of the Case, 6 octobre 2009.

49. Idem, paragraphe 9. 
perplexe: comment un droit si fondamental, si essentiel dans la procédure devant la Cour d'après les affirmations répétées des différentes chambres, pourrait être limité à la simple demande du greffe par exemple?

La Chambre décidait en l'espèce de ne donner accès aux victimes qu'aux audiences publiques, aux documents et décisions publics ainsi qu'aux éléments de preuve publics. Comme le procureur décidait d'appeler trois témoins à l'audience de confirmation des charges, la Chambre précisait que les représentants légaux devraient présenter une requête spécifique s'ils souhaitaient interroger un de ces témoins et qu'elle déciderait alors. Les représentants légaux des victimes se voyaient également octroyer le droit de présenter des conclusions écrites ou orales, la Chambre n'exigeant pas que ces conclusions soient brèves. Cette jurisprudence a été maintenue par la Chambre préliminaire I dans l'affaire Banda et Jerbo ${ }^{50}$.

\section{Le rôle des victimes au stade du procès}

C'est au stade du procès que l'on constate une certaine avancée des droits procéduraux des victimes, dans des limites modestes cependant. Les chambres de première instance ont été confrontées, comme les chambres préliminaires, aux questions relatives aux limites de l'intervention des victimes, à l'établissement d'un système pour la participation des victimes et enfin à la définition des modalités de participation des victimes.

La première question est bien entendu, comme au stade préliminaire de l'affaire, la question des limites de l'intervention des victimes, et là encore la réponse a été très claire: puisque, selon la Chambre d'appel, «les paramètres énoncés dans les charges définissent les questions à trancher lors du procès et limitent l'autorité de la Chambre de première instance à l'examen de ces questions ${ }^{51} »$, l'intervention des victimes lors du procès a pour limite les charges telles que confirmées par la Chambre

50. CPI, Situation in Darfur, Sudan, In the Case of the Prosecutor v. Abdallab Banda Abakaer Nourain and Saleb Mohammed Jerbo Jamus, ICC-02/05-03/09-89, Pre-Trial Chamber I, Decision on Victims' Participation at the Hearing on the Confirmation of the Charges, 29 octobre 2010.

51. CPI, Situation en République démocratique du Congo, Affaire le Procureur c. Thomas Lubanga Dyilo, ICC-01/04-01/06-1432-tFRA, Chambre d'appel, Arrêt relatif aux appels interjetés par le Procureur et la Défense contre la Décision relative à la participation des victimes rendue le 18 janvier 2008 par la Chambre de première instance I, 11 juillet 2008, paragraphe 63. 
préliminaire dans sa décision prise en application de l'article 61, paragraphe 7, du Statut.

Cependant si l'article 74, paragraphe 2, du Statut précise que la décision de la Chambre de première instance ne peut aller au-delà des faits et des circonstances décrits dans les charges et les modifications apportées à celles-ci, la norme 55 du Règlement de la Cour permet à la Chambre de première instance, dans la limite des faits décrits dans les charges et les modifications apportées à celles-ci, de modifier la qualification juridique des faits.

Le 22 mai $2009^{52}$, les représentants légaux des victimes sollicitaient donc de la Chambre de première instance I qu'elle mette en œuvre la procédure prévue à la norme $55 \mathrm{du}$ Règlement de la Cour. Alors que les charges retenues contre Thomas Lubanga Dyilo concernaient uniquement le fait de procéder à la conscription et à l'enrôlement d'enfants de moins de 15 ans dans les forces armées nationales et de les faire participer activement à des hostilités (faits prévus et réprimés par les articles 8 , paragraphe $2 \mathrm{~b}$ ] xxvi], dans le cadre d'un conflit armé international et 8 , paragraphe 2 e] vii], dans le cadre d'un conflit armé non international, du Statut), les représentants légaux des victimes soulignaient dans leur requête que les faits relatés par un certain nombre de témoins qui avaient déposé devant la Chambre se rapportaient aux éléments de crimes pouvant tomber dans les catégories prévues aux articles 8, paragraphe 2 a) ii) («traitement inhumain») ou 8, paragraphe 2 c) i) («traitements cruels») du Statut et 7, paragraphe $1 \mathrm{~g}$ ) ou 8, paragraphe 2 b) xxii) ou 8, paragraphe 2 e) vi) («esclavage sexuel») du Statut.

La défense ${ }^{53}$ s'opposait à cette demande qui, selon elle, sous couvert de requalification juridique des faits, visait en réalité à ajouter d'autres infractions à celles figurant dans la décision de confirmation des charges et aurait pour conséquence d'ajouter aux faits et circonstances décrits dans les charges.

52. CPI, Situation en République démocratique du Congo, Affaire le Procureur c. Thomas Lubanga Dyilo, ICC-01/04-01/06-1891, représentants légaux des victimes, Demande conjointe des représentants légaux des victimes aux fins de mise en œuvre de la procédure en vertu de la norme 55 du Règlement de la Cour, 22 mai 2009.

53. CPI, Situation en République démocratique du Congo, Affaire le Procureur c. Thomas Lubanga Dyilo, ICC-01/04-01/06-1975, équipe de la défense de Thomas Lubanga Dyilo, Réponse de la Défense à la «Demande conjointe des représentants légaux des victimes aux fins de mise en œuvre de la procédure en vertu de la norme 55 du Règlement de la Cour» datée du 22 mai 2009 et à la «Prosecution's response to the legal representatives "Demande conjointe des représentants légaux des victimes aux fins de mise en œuvre de la procédure en vertu de la norme 55 du Règlement de la Cour" » datée du 12 juin 2009, 19 juin 2009. 
Le 14 juillet $2009^{54}$, la majorité (à savoir les juges Elizabeth Odio Benito et René Blattmann) de la Chambre de première instance I faisait droit à la requête des victimes et estimait que la procédure prévue à la norme 55 du Règlement de la Cour pouvait être mise en œuvre, tout en renvoyant à plus tard son application effective. La majorité de la Chambre estimait en effet que la procédure visant à modifier la qualification juridique des faits en application de la norme 55 pouvait aboutir à englober de nouveaux faits dans la prévention.

Saisie par le procureur et la défense, la Chambre d'appel, dans une décision du 8 décembre $2009^{55}$, infirmait la décision de la Chambre de première instance. La Chambre d'appel précisait en effet que si la Chambre de première instance pouvait modifier la qualification juridique des faits décrits dans les charges, elle ne pouvait en aucun cas aller au-delà des faits et circonstances décrits dans les charges. Par faits et circonstances décrits dans les charges, la Chambre d'appel indiquait ${ }^{56}$ qu'il fallait comprendre les «allégations factuelles étayant chacun des éléments juridiques du crime faisant l'objet des charges» à l'exclusion «des informations éclairant le contexte et autres informations générales, qui bien qu'elles figurent dans le document de notification des charges ou dans la décision relative à la confirmation des charges, n'étayent pas les éléments juridiques du crime faisant l'objet des charges». Il était donc impossible pour la Chambre de première instance, contrairement à ce que soutenaient les représentants légaux des victimes, de prendre en compte les témoignages lors du procès pour ajouter des faits à ceux décrits dans la décision de confirmation des charges.

S'agissant de la deuxième question, les systèmes retenus par les chambres de première instance dans les procès qui se déroulent devant la CPI diffèrent puisque les Chambres de première instance I et III ont retenu pour l'appréciation du critère, prévu à l'article 68, paragraphe 3, du Statut, des intérêts personnels des victimes, une approche au cas par

54. CPI, Situation en République démocratique du Congo, Affaire le Procureur c. Thomas Lubanga Dyilo, ICC-01/04-01/06-2049-tFRA, Chambre de première instance I, Décision informant les parties et les participants que la qualification juridique des faits peut être modifiée conformément à la norme 55-2 du Règlement de la Cour, 14 juillet 2009.

55. CPI, Situation en République démocratique du Congo, Affaire le Procureur c. Thomas Lubanga Dyilo, ICC-01/04-01/06-2205-tFRA, Chambre d'appel, Arrêt relatif aux appels interjetés par Thomas Lubanga Dyilo et par le Procureur contre la Décision informant les parties et participants que la qualification juridique des faits peut être modifiée conformément à la norme 55-2 du Règlement de la Cour, 8 décembre 2009.

56. Idem, paragraphe 90 et note de bas de page $\mathrm{n}^{\circ} 163$. 
cas, alors que la Chambre de première instance II a retenu une approche plus systématique.

En effet, les Chambres de première instance $\mathrm{I}^{57}$ et $\mathrm{III}^{58}$ ont précisé que «la participation des victimes ne s'apprécie pas une fois pour toutes mais qu'elle doit être décidée au regard des preuves ou des questions examinées à un moment précis». Ainsi, les victimes qui sont reconnues en tant que victimes dans une affaire donnée car elles ont subi un préjudice du fait des crimes contenus dans les charges confirmées contre l'accusé ont un droit général à participer à la procédure mais ce droit général est dépourvu de contenu puisque pour chaque intervention procédurale, écrite ou orale, le représentant légal de ces victimes devra de nouveau prouver en quoi les intérêts personnels des victimes qu'il représente justifient son intervention à un moment donné du procès.

Ce système peut s'avérer complexe puisque le représentant légal des victimes doit justifier chacune de ses interventions: plus qu'un droit de participer au procès, c'est plutôt un droit d'assister au procès, le droit d'intervenir étant soumis à une autorisation spécifique et limitée dans le temps à la partie du procès ou à la question soulevée à un moment donné pour laquelle l'intervention est sollicitée et autorisée.

La Chambre de première instance $\mathrm{II}^{59}$ a expressément refusé de suivre cette approche en décidant qu'elle n'exigerait pas des représentants légaux des victimes qu'ils déposent une demande écrite justifiant au préalable chacune de leurs interventions: ceux-ci seront présumés intervenir au nom du groupe de victimes qu'ils représentent. En d'autres termes, l'intérêt personnel des victimes est un intérêt à participer au

57. CPI, Situation en République démocratique du Congo, Affaire le Procureur c. Thomas Lubanga Dyilo, ICC-01/04-01/06-1119-tFRA, Chambre de première instance I, Décision relative à la participation des victimes, 18 janvier 2008, paragraphes 96, 101 et 103 .

58. CPI, Situation in the Central African Republic, in the Case of the Prosecutor v. Jean-Pierre Bemba Gombo, ICC-01/05-01/08-807, Trial Chamber III, Decision on the participation of victims in the trial and on 86 applications by victims to participate in the proceedings (uniquement disponible en anglais), 30 juin 2010, paragraphe 25. Si la Chambre de première instance III a suivi le système établi par la Chambre de première instance I, c'est probablement parce qu'au moment où la Chambre de première instance III a pris sa décision sur la participation des victimes, deux des trois juges de la Chambre de première instance I qui avaient pris la décision du 18 janvier 2008 faisaient également partie de la Chambre de première instance III (les juges Fulford et Odio Benito).

59. CPI, Situation en République démocratique du Congo, Affaire le Procureur c. Germain Katanga et Matbieu Ngudjolo Chui, ICC-01/04-01/07-1788, Chambre de première instance II, Décision relative aux modalités de participation des victimes au stade des débats sur le fond, 22 janvier 2010, paragraphes 61 et 63. 
procès dans son ensemble et cet intérêt n'a pas à être démontré au cas par cas pour chaque intervention spécifique de son représentant légal:

La Chambre considère que les intérêts personnels de chacune des victimes prises individuellement ont donc été démontrés et que leurs représentants légaux n'auront pas, au cours des débats sur le fond, à renouveler cette démonstration. En d'autres termes, dès lors que la Chambre a décidé qu'une victime donnée pouvait participer à la procédure conformément à la règle 89 du Règlement, cela signifie qu'elle lui a reconnu par là même un intérêt personnel en l'espèce.

S'il s'agit de l'approche générale de la Chambre de première instance II au regard de l'appréciation du critère des intérêts personnels des victimes, la Chambre n'en demande pas moins, comme on le verra, aux représentants légaux de solliciter à l'avance l'autorisation de la Chambre lorsqu' ils veulent effectuer certains actes procéduraux comme interroger un témoin, conformément à la règle 91 du Règlement, ou solliciter l'autorisation de présenter des preuves. En pratique donc, même si les systèmes institués par les chambres de première instance apparaissent différents, leur application semble aboutir pour les représentants légaux des victimes à un résultat assez similaire.

En ce qui concerne la troisième question, à savoir celle des modalités de participation des victimes, les chambres de première instance ont accordé des droits différents en fonction des procès, conformément à ce qu'elles estiment être leur pouvoir discrétionnaire en la matière.

La Chambre de première instance I, suivie de la Chambre de première instance III, ayant adopté un système d'intervention au cas par cas, s'est contentée de fixer des directives générales ${ }^{60}$. Ainsi, elle a donné le droit aux représentants légaux des victimes de consulter le dossier, les pièces et les écritures à condition qu'ils soient publics, tout comme le droit de participer aux audiences publiques. Pour la participation aux audiences à huis clos ou l'accès aux documents confidentiels, la Chambre s'est réservé le droit de décider, au cas par cas, si les intérêts des victimes représentées justifiaient une telle participation ou un tel accès ${ }^{61}$. Elle a également donné le droit aux représentants légaux des victimes de contester l'admissibilité ou la pertinence des preuves présentées lorsque les intérêts personnels des victimes étaient concernés ${ }^{62}$.

60. CPI, Situation en République démocratique du Congo, Affaire le Procureur c. Thomas Lubanga Dyilo, ICC-01/04-01/06-1119-tFRA, Chambre de première instance I, Décision relative à la participation des victimes, 18 janvier 2008, paragraphe 84 .

61. Idem, paragraphes 105 et 113.

62. Ibidem, paragraphe 109. 
Un aspect particulièrement contentieux de la décision de la Chambre de première instance I était la possibilité reconnue aux victimes de présenter des éléments de preuve concernant la responsabilité pénale de l'accusé ${ }^{63}$. Cet aspect de la décision a fait l'objet d'une demande d'autorisation d'appel de la part du procureur et de la défense, que la Chambre décidait d'accepter.

Le procureur et la défense ont soutenu devant la Chambre d'appel qu'une telle prérogative ne pouvait appartenir qu'aux parties à la procédure, à savoir le procureur et la défense. La Chambre d'appel ${ }^{64}$ a rejeté les arguments du procureur et de la défense et a confirmé la décision de la Chambre de première instance I sur ce point, mais à une très courte majorité puisque l'arrêt de la Chambre d'appel a été adopté par trois voix contre deux. Cet arrêt de la Chambre d'appel a constitué une victoire pour les droits des victimes. La Chambre d'appel a estimé en effet que l'article 69, paragraphe 3, du Statut, lu conjointement avec l'article 68 , paragraphe 3 , du Statut et la règle 91, paragraphe 3 , du Règlement, laissait ouverte la possibilité pour les victimes de solliciter de la Chambre de première instance l'autorisation de présenter des éléments de preuve, si la Chambre estimait que cela était nécessaire pour la détermination de la vérité. La Chambre d'appel a considéré en effet:

Pour que l'esprit et l'intention de l'article 68-3 du Statut se traduisent dans les faits lors du procès, cet article doit être interprété de manière à ce que la participation des victimes soit significative. Des éléments de preuve soumis lors du procès qui seraient sans rapport avec la culpabilité ou l'innocence de l'accusé seraient très vraisemblablement considérés comme irrecevables ou sans intérêt. Si les victimes se voyaient de manière générale et en toutes circonstances dans l'impossibilité de présenter des éléments de preuve touchant à la culpabilité ou à l'innocence de l'accusé et de contester l'admissibilité ou la pertinence d'autres preuves, leur droit de participer au procès pourrait devenir sans effet ${ }^{65}$.

Mais la possibilité pour les victimes de soumettre des éléments de preuve a été strictement encadrée puisqu'elle a été soumise à l'autorisation de la Chambre de première instance statuant sur requête présentée par les

63. Ibidem, paragraphe 108.

64. CPI, Situation en République démocratique du Congo, affaire le Procureur c. Thomas Lubanga Dyilo, ICC-01/04-01/06-1432-tFRA, Chambre d'appel, Arrêt relatif aux appels interjetés par le Procureur et la Défense contre la Décision relative à la participation des victimes rendue le 18 janvier 2008 par la Chambre de première instance I, 11 juillet 2008.

65. Idem, paragraphe 97. 
représentants légaux des victimes. Une telle procédure laisse bien entendu la possibilité au procureur et à la défense de répondre, et éventuellement de s'opposer, à la requête des représentants légaux des victimes, qui devront exposer en quoi leurs intérêts personnels sont concernés.

La possibilité pour les représentants légaux des victimes de présenter des preuves n'est donc pas, pour l'instant dans la jurisprudence de la CPI, un droit procédural autonome des victimes mais simplement une façon d'inviter la Chambre à user de son pouvoir, prévu à l'article 69, paragraphe 3, du Statut, qui lui permet de solliciter la présentation des preuves qu'elle estime nécessaires à la manifestation de la vérité. La source procédurale de cette possibilité reconnue aux représentants légaux n'est donc pas vraiment l'article 68, paragraphe 3, du Statut, mais plutôt l'article qui définit les pouvoirs de la Chambre, à savoir l'article 69, paragraphe 3, du Statut.

La Chambre d'appel a rappelé ce principe dans un arrêt de juillet 2010 où elle insiste sur le fait que les victimes n'ont pas le droit de présenter des éléments de preuve mais simplement la possibilité d'inviter la Chambre de première instance à user du pouvoir discrétionnaire que lui donne l'article 69, paragraphe 3, du Statut de demander la présentation de tous les éléments de preuve qu'elle juge nécessaires à la manifestation de la vérité ${ }^{66}$.

Pour inviter la Chambre de première instance à user de ce pouvoir, les représentants légaux des victimes doivent en outre prouver que les conditions prévues à l'article 68, paragraphe 3, du Statut sont réunies, à savoir que les intérêts personnels des victimes qu'ils représentent sont concernés, que la présentation de cette preuve est appropriée et ne serait pas contraire aux droits de la défense et aux exigences d'un procès équitable et impartial. On peut se demander pourquoi la Chambre d'appel a tant d'exigences alors qu'il s'agit de tenter de convaincre la Chambre de première instance d'utiliser un pouvoir qu'elle peut de toute façon utiliser de sa propre initiative. Ainsi, une ONG, sous l'angle de la règle $103 \mathrm{du}$ Règlement ${ }^{67}$, pourrait suggérer à la Chambre de pre-

66. CPI, Situation en République démocratique du Congo, Affaire le Procureur c. Germain Katanga et Matbieu Ngudjolo Chui, ICC-01/04-01/07-2288-tFRA, Chambre d'appel, Arrêt relatif à l'appel interjeté par Germain Katanga contre la Décision relative aux modalités de participation des victimes au stade des débats sur le fond, rendue le 22 janvier 2010 par la Chambre de première instance II, 16 juillet 2010, paragraphes 40 et 48 .

67. Règle 103, Amicus curice et autres formes de déposition. La règle 103(1) est ainsi rédigée: À n'importe quelle phase de la procédure, toute chambre de la Cour peut, si elle le juge souhaitable en l'espèce pour la bonne administration de la justice, inviter ou 
mière instance d'user de son pouvoir discrétionnaire sans avoir à remplir les exigences de l'article 68, paragraphe 3, du Statut.

C'est là une limitation sévère des droits des victimes au cours du procès puisque ce droit est limité à la possibilité de suggérer à la Chambre l'utilisation de ses propres pouvoirs. Pour aboutir à une participation réellement effective des victimes à la procédure, il faudrait reconnaître comme source de leurs droits procéduraux l'article 68, paragraphe 3, du Statut et ne pas soumettre l'exercice de leurs droits au bon vouloir des juges. Les juges devraient avoir comme seule prérogative, au demeurant essentielle, d'examiner les preuves présentées par les représentants légaux des victimes, au même titre qu'ils examinent les preuves soumises par le procureur ou la défense.

Il est possible que les juges aient voulu éviter la critique d'une interprétation extensive de l'article 68, paragraphe 3, du Statut concernant les droits des victimes en justifiant cette possibilité laissée aux victimes de présenter des preuves par l'utilisation de leurs propres pouvoirs sous l'angle de l'article 69, paragraphe 3, du Statut.

Malgré ces limitations, la Chambre de première instance I a autorisé, en $2009^{68}$, le représentant légal des victimes à présenter trois des victimes qu'il représentait comme témoins au procès.

La Chambre de première instance en a profité pour rappeler, contrairement à ce qu'affirmait le procureur, que la Chambre d'appel, dans son arrêt du 11 juillet 2008, n'a jamais exigé que les victimes démontrent des circonstances exceptionnelles avant de pouvoir soumettre des preuves à la Chambre. En revanche, une telle procédure suppose que les victimes deviennent des témoins qui devront prêter serment et seront soumis aux questions du procureur, de la défense et des représentants légaux des victimes. De même, les représentants légaux des victimes devront présenter un procès-verbal de ces témoignages avant la com-

autoriser tout État, toute organisation ou toute personne à présenter par écrit ou oralement des observations sur toute question qu'elle estime appropriée.

68. CPI, Situation in the Democratic Republic of the Congo, In the Case of the Prosecutor $v$. Thomas Lubanga Dyilo, Trial Chamber I, Decision on the request by victims a/0225/06, a/0229/06 and a/0270/07 to express their views and concerns in person and to present evidence during the trial; cette décision est une décision confidentielle du 26 juin 2009 dont la version publique expurgée est en annexe d'une décision rendue le 9 juillet 2009 intitulée: «Order issuing public redacted version of the "Decision on the request by victims a/0225/06, a/0229/06 and a/0270/07 to express their views and concerns in person and to present evidence during the trial"»; ces décisions sont disponibles en anglais uniquement et portent les numéros ICC-01/04-01/06-2032 et ICC-01/04-01/06-2032Anx. 
parution des victimes devant la Chambre pour que le procureur et la défense puissent se préparer.

La Chambre de première instance II ayant, elle, adopté une approche plus systématique de la participation des victimes à la procédure, le système adopté est plus précis et plus développé (environ 60 paragraphes et 20 pages!) ${ }^{69}$.

La Chambre a reconnu tout d'abord aux représentants légaux des victimes le droit d'assister non seulement aux audiences publiques, mais également aux audiences à huis $\operatorname{clos}^{70}$, contrairement à ce qu'avait décidé la Chambre de première instance I qui avait, en principe, limité la participation des représentants légaux aux audiences publiques, sauf autorisation donnée au cas par cas.

La Chambre créait ensuite la possibilité pour les représentants légaux d'interroger les témoins, les experts ou même les accusés (sous réserve bien entendu du droit pour ces derniers de ne faire aucune déclaration). Sur ce point, elle avait déjà précisé dans une décision prise en $2009^{71}$ que les représentants légaux désirant interroger un témoin, un expert ou les accusés sur des points ne se rapportant pas aux réparations, devaient en informer la Chambre par une demande écrite au moins sept jours avant la comparution du témoin. Cette requête devait indiquer les questions que le représentant légal se proposait de poser et expliquer en quoi ces questions se rapportaient aux intérêts personnels des victimes qu'il représentait. Elle précisait que le représentant légal serait autorisé à poser les questions après l'interrogatoire principal mené par la partie qui avait cité le témoin, à moins que la Chambre décide ellemême de poser ces questions au nom du représentant légal, conformément à ce qui était prévu à la règle 91(3) du Règlement.

La Chambre ${ }^{72}$ précisait également la façon dont les représentants légaux devaient conduire leurs interrogatoires. Ainsi elle précisait tout d'abord, suivant en cela la jurisprudence de la Chambre de première

69. CPI, Situation en République démocratique du Congo, Affaire le Procureur c. Germain Katanga et Mathieu Ngudjolo Chui, ICC-01/04-01/07-1788, Chambre de première instance II, Décision relative aux modalités de participation des victimes au stade des débats sur le fond, 22 janvier 2010, paragraphes 65 à 125 .

70. Idem, paragraphe 71 .

71. CPI, Situation en République démocratique du Congo, Affaire le Procureur c. Germain Katanga et Matbieu Ngudjolo Chui, ICC-01/04-01/07-1665-Corr-tFRA, Chambre de première instance II, Instructions pour la conduite des débats et les dépositions conformément à la règle $140,1^{\text {er }}$ décembre 2009 , paragraphes 87 et 88 .

72. Idem, paragraphe 91; voir aussi CPI, Situation en République démocratique du Congo, Affaire le Procureur c. Germain Katanga et Matbieu Ngudjolo Chui, ICC-01/04-01/07-1788, 
instance $\mathrm{I}^{73}$, que le but d'un tel interrogatoire devait être la manifestation de la vérité, «les victimes n'étant pas des parties au procès et n'ayant pas pour rôle de soutenir la cause du Procureur».

Là encore, on aperçoit un glissement de l'article 68, paragraphe 3, $\mathrm{du}$ Statut, qui vise les droits des victimes, vers l'article 69, paragraphe 3, du Statut qui vise les pouvoirs de la Chambre de solliciter la présentation des éléments de preuve qu'elle estime nécessaires à la manifestation de la vérité. Les représentants légaux des victimes deviennent des auxiliaires de la Chambre dans sa recherche de la vérité ou sont à tout le moins présentés comme tels.

On ne trouve pourtant une telle condition à l'exercice des droits procéduraux des victimes, ni à l'article 68 , paragraphe $3 \mathrm{du}$ Statut, ni à la règle $91 \mathrm{du}$ Règlement. En effet, l'article 68, paragraphe 3, du Statut oblige la Cour à permettre aux victimes de présenter leurs vues et préoccupations, et celles-ci peuvent ne pas forcément correspondre à la recherche de la vérité, telle que l'entend la Chambre. Une telle interprétation tend à faire de la participation des victimes un instrument aux mains des juges, qu'ils peuvent utiliser comme ils le souhaitent. De victimes utilisées par le procureur dans les deux tribunaux ad hoc pour l'ex-Yougoslavie et le Rwanda pour prouver «ses affaires», il faudrait éviter de passer à des victimes utilisées par les juges, à la CPI, dans la recherche de ce qu'ils estiment nécessaire à la manifestation de la vérité.

La Chambre insistait également sur le fait que les représentants légaux des victimes ne devraient pas suivre les techniques du contreinterrogatoire qui peuvent consister à poser des questions fermées, directives ou provocatrices, mais plutôt suivre un mode d'interrogatoire neutre, à moins que la Chambre ne les autorise à utiliser un autre mode d'interrogatoire.

S'agissant de la possibilité pour les représentants légaux des victimes de présenter des preuves, la Chambre faisait ${ }^{74}$ une distinction entre la

Chambre de première instance II, Décision relative aux modalités de participation des victimes au stade des débats sur le fond, 22 janvier 2010, paragraphes 75 et 78 .

73. CPI, Situation en République démocratique du Congo, Affaire le Procureur c. Thomas Lubanga Dyilo, ICC-01/04-01/06-2127-tFRA, Chambre de première instance I, Décision relative aux modes d'interrogation des témoins par les représentants légaux des victimes, 16 septembre 2009, paragraphes 24 à 30 .

74. CPI, Situation en République démocratique du Congo, Affaire le Procureur c. Germain Katanga et Matbieu Ngudjolo Chui, ICC-01/04-01/07-1788, Chambre de première instance II, Décision relative aux modalités de participation des victimes au stade des débats sur le fond, 22 janvier 2010, paragraphes 85 à 97. 
possibilité d'appeler les victimes qu'ils représentaient à comparaître en tant que témoins, et la possibilité de suggérer d'autres témoins: si les représentants légaux se sont vu reconnaitre «la possibilité de citer une ou plusieurs victimes en vue de témoigner sous serment au procès», en revanche, ils ne peuvent que suggérer à la Chambre les noms d'autres témoins éventuels que la Chambre décidera ou non de citer en application du pouvoir qu'elle tient de l'article 69, paragraphe 3, du Statut. La Chambre a effectivement autorisé des victimes à témoigner devant $e \mathrm{ell}^{75}$, à la demande de leur représentant légal, comme l'avait déjà fait la Chambre de première instance I dans l'affaire Thomas Lubanga Dyilo.

La Chambre laissait également la possibilité aux représentants légaux des victimes, avec l'autorisation de la Chambre, de déposer des preuves documentaires $^{76}$, à condition encore une fois que cela puisse l'assister dans la mise en œuvre de l'article 69, paragraphe 3, du Statut, et par là même dans $s a$ recherche de la vérité.

La Chambre permettait également aux représentants légaux des victimes de contester l'admissibilité ou la pertinence d'un élément de preuve $^{77}$, déposé soit par le procureur, soit par la défense.

Enfin la Chambre de première instance II, contrairement à la Chambre de première instance $\mathrm{I}$, donnait accès aux représentants légaux des victimes à la totalité des décisions et documents publics ou confidentiels ${ }^{78}$ figurant au dossier de l'affaire, et à tous les éléments de preuve produits par le procureur ou la défense ${ }^{79}$. Sur cette question très sensible de l'accès aux décisions et documents confidentiels ainsi qu'aux éléments de preuve, la Chambre de première instance III $^{80}$ a tenté de

75. CPI, Situation en République démocratique du Congo, Affaire le Procureur c. Germain Katanga et Matbieu Ngudjolo Chui, ICC-01/04-01/07-2517, Chambre de première instance II, Décision aux fins d'autorisation de comparution des victimes a/0381/09, a/0018/09, a/0191/08 et pan/0363/09 agissant au nom de a/0363/09, 9 novembre 2010.

76. CPI, Situation en République démocratique du Congo, Affaire le Procureur c. Germain Katanga et Mathieu Ngudjolo Chui, ICC-01/04-01/07-1788, Chambre de première instance II, Décision relative aux modalités de participation des victimes au stade des débats sur le fond, 22 janvier 2010, paragraphe 98.

77. Idem, paragraphe 104 .

78. Ibidem, paragraphe 121 .

79. Ibidem, paragraphe 122 .

80. CPI, Situation in the Central African Republic, in the Case of the Prosecutor v. Jean-Pierre Bemba Gombo, ICC-01/05-01/08-807, Trial Chamber III, Decision on the participation of victims in the trial and on 86 applications by victims to participate in the proceedings (uniquement disponible en anglais), 30 juin 2010, paragraphes 47 à 49 . 
parvenir à un compromis en décidant que les représentants légaux devaient avoir accès seulement aux décisions et documents publics, mais en établissant elle-même au même moment une liste de documents confidentiels que les représentants légaux devaient obtenir avant le début du procès. Cette chambre a enfin décidé que les procès-verbaux des témoignages de tous les témoins que le procureur entendait faire comparaître devaient également être communiqués aux représentants légaux des victimes.

Là encore, mais dans une moindre mesure que pour les victimes de la situation, la jurisprudence de la CPI est restrictive en ce qui concerne les droits des victimes de l'affaire et ne fait pas à ces victimes la place qui leur permettrait de réellement influer sur la procédure devant la Cour plutôt que d'avoir la désagréable impression d'assister à un débat judiciaire dans lequel elles demeurent largement étrangères. Pour pourvoir agir, les victimes de l'affaire, tout comme les victimes de la situation, doivent d'abord et avant tout, avoir accès à l'information, à savoir ici avoir accès aux éléments de preuve qui forment la base du débat judiciaire. Rien dans les textes actuels ne justifie la jurisprudence extrêmement restrictive particulièrement des chambres préliminaires qui les maintient dans l'ignorance du dossier de l'affaire et donc rend leur participation artificielle. En ce qui concerne leur droit d'agir, il conviendrait de leur permettre de présenter les éléments de preuve nécessaires à la défense de leurs intérêts, dans le respect bien entendu des droits de la défense, et sans laisser l'exercice de ce droit à la totale discrétion des juges.

\section{Conclusion}

Si l'innovation concernant la participation des victimes à la procédure était présentée comme fondamentale en 1998, sa mise en œuvre par la jurisprudence laisse un sentiment d'incertitude aux victimes.

On pouvait s'y attendre puisque le texte qui a inspiré l'article 68 , paragraphe 3, du Statut, à savoir le paragraphe 6 b) de l'Annexe à la Déclaration des principes fondamentaux de justice relatifs aux victimes de la criminalité et aux victimes d'abus de pouvoir ${ }^{81}$, était un texte de compromis qui laissait aux différents pays la possibilité d'une application

81. Précitée, Résolution $n^{\circ} 40 / 34$ adoptée par l'Assemblée générale des Nations Unies le 29 novembre 1985. 
fort différente de cette Déclaration en fonction de leur tradition juridique ${ }^{82}$.

Si on peut comprendre un tel compromis, et donc une telle imprécision dans les droits réellement accordés aux victimes, dans un texte qui n'est qu'un guide pour des pays aux cultures juridiques fort différentes, il est plus difficile d'accepter une telle imprécision au sein d'une même Cour. Ceci est peut-être la preuve qu'il ne faut pas forcément s'inspirer de ce qui existe déjà dans la sphère internationale pour la négociation d'autres textes internationaux, même si les diplomates ont toujours tendance, pour faciliter les discussions, à utiliser ce qu'ils appellent en anglais «agreed language».

La solution, pour une meilleure intégration des victimes à la procédure devant la CPI, devrait passer par une codification des droits des victimes même si la discussion peut se révéler très délicate au sein de l'Assemblée des États Parties: si la modification du Statut semble très difficile eu égard aux exigences de ratification de tout amendement au Statut par les 7/8 des États Parties en application de l'article $121 \mathrm{du}$ Statut, on pourrait au moins envisager une amorce de codification de certains droits procéduraux des victimes dans le Règlement, dont la modification dépend, conformément à l'article $51 \mathrm{du}$ Statut, de la seule décision de l'Assemblée des États Parties et qui ne nécessite pas une ratification par les États Parties.

Cette codification devrait d'abord permettre de préciser le droit et les modalités permettant aux victimes d'avoir accès à des informations précises sur les enquêtes et affaires en cours dans des délais stricts. Ceci est particulièrement crucial pour les victimes de la situation lorsque le procureur décide de ne pas enquêter ou de ne pas poursuivre.

En ce qui concerne les victimes de l'affaire, leur participation devrait être précisée et renforcée, notamment au stade de la confirmation des charges, puisque c'est à ce moment de la procédure qu'est définitivement fixée l'étendue des charges retenues contre la personne accusée et donc le «cadre du procès». Les victimes devraient par exemple pouvoir à ce stade présenter des preuves ou solliciter des juges qu'ils ordonnent un complément d'enquête avant le renvoi de l'affaire en procès lorsqu'elles

82. Voir sur ce point: Handbook on Justice For victims on the use and application of the Declaration of Basic Principles of Justice for Victims of Crime and Abuse of Power, United Nations Office for Drug Control and Crime Prevention, Centre for International Crime Prevention, New York, 1999, pages 36 à 39. 
estiment que le procureur n'a pas présenté suffisamment d'éléments pour établir la réalité des faits commis.

De manière plus générale, les victimes de l'affaire devraient pouvoir, sans nécessairement être soumises à la discrétion des juges, participer à l'établissement de la vérité en apportant des preuves sur la véritable nature des crimes commis: sur ce point, la qualification des faits et l'ampleur des crimes subis peuvent être un aspect essentiel pour les victimes qui doivent avoir les moyens procéduraux d'apporter des preuves établissant, par exemple, qu'un génocide a été commis alors même que le procureur trouverait plus simple d'établir l'existence de crimes de guerre ou de crimes contre l'humanité. Bien entendu, la participation des victimes doit respecter les droits de la défense, ce qui signifie que tout élément apporté par les victimes au débat judiciaire devrait être soumis à la défense pour une discussion contradictoire.

ABSTRACT - While the introduction on procedural rights for victims before the International Criminal Court is undoubtedly a fundamental change for the two Ad-hoc tribunals for the Former-Yugoslavia and Rwanda, the innovation is characterised by imprecision. In this article we will study victims' right to participate in procedures. We will first study the procedural right of victims of the situation and then the rights of victims of the case before the courts still contains much uncertainty for victims. The solution for a better integration of victims in the procedures before the ICC would be the codification of victims' rights.

KEYWORDS - Victims' rights, participation, International Criminal Court, international law, judicial review, prosecutorial discretion.

RESUMEN - Cambio fundamental sin duda respecto de los tribunales ad hoc constituidos para la ex Yugoslavia y Ruanda, el establecimiento de derechos procesales para las víctimas en la Corte Penal Internacional es todavía una innovación imprecisa. En este artículo se estudian los derechos a participar en los procedimientos penales, primero en el caso de las víctimas de una situación general, y posteriormente, en el de las víctimas de incidentes específicos. El artículo concluye que la puesta en marcha de la participación de las víctimas por vía de la jurisprudencia les deja aún un sentimiento de incertidumbre. La solución para su mejor integración en los procesos de la CPI es que la participación de las víctimas se dé mediante la codificación de sus derechos.

PALABRAS CLAVE - Derechos de las víctimas, participación, Corte penal internacional, derecho internacional, control judicial, poder discrecional del procurador. 